\title{
Assessing gridded observations for daily precipitation extremes in the Alps with a focus on northwest Italy
}

\author{
M. Turco ${ }^{1}$, A. L. Zollo ${ }^{1}$, C. Ronchi ${ }^{2}$, C. De Luigi ${ }^{2}$, and P. Mercogliano ${ }^{1}$ \\ ${ }^{1} \mathrm{CMCC}$ (Euro-Mediterranean Centre on Climate Change), Impacts on Soil and Coasts Division, via Maiorise s.n.c., \\ 81043 Capua (CE), Italy \\ ${ }^{2}$ Arpa Piemonte, Dipartimento Sistemi Previsionali, via Pio VII, 9 - 10135 Torino, Italy
}

Correspondence to: M. Turco (marco.turco@cmcc.it)

Received: 31 October 2012 - Published in Nat. Hazards Earth Syst. Sci. Discuss.: Revised: 23 April 2013 - Accepted: 28 April 2013 - Published: 11 June 2013

\begin{abstract}
In this study we compare three gridded observed datasets of daily precipitation (EOBS, MAP and NWIOI) over the Great Alpine Region (GAR) and a subregion in northwest Italy (NWI) in order to better understand the past variability of daily climate extremes and to set up a basis for developing regional climate scenarios. The grids are first compared with respect to their temporal similarity by calculating the correlation and relative mean absolute error of the time series. They are then compared with respect to their spatial similarity to the climatology of the ETCCDI indices (characterizing total precipitation, dry and wet spells and extremes with short return periods). The results indicate first that most EOBS gridpoint series in northeastern Italy have to be shifted back by 1 day to have maximum overlap of the measurement period and, second, that both the temporal and spatial similarities of most indices are higher between the NWIOI and MAP than between MAP or the NWIOI and EOBS. These results suggest that, although there is generally good temporal agreement between the three datasets, EOBS should be treated with caution, especially for extreme indices over the GAR region, and it does not provide reliable climatology over the NWI region. The high agreement between MAP and NWIOI, on the other hand, builds confidence in using these datasets. Users should consider carefully the limitations of the gridded observations available: the uncertainties of the observed datasets cannot be neglected in the overall uncertainties cascade that characterizes climate change studies.
\end{abstract}

\section{Introduction}

The European Alpine region, which is geographically complex and heterogeneous, is characterized by a great variability of precipitation regimes (Schär et al., 1998) due to the influences of different climatological regimes, such as the Mediterranean, continental, Atlantic, and polar ones (Beniston, 2005). In addition, precipitation plays a major role in water resources and natural hazards in this area, with high hydrogeological risk and strong human pressure (Bates et al., 2008).

As a first step in developing high-resolution regional climate scenarios for climate change impact/adaptation studies, it is necessary to carefully analyse long observation datasets, especially those data which allow us to focus on precipitation extremes on a regional scale. Unfortunately, a number of constraints, such as periods of unavailability and poorquality data, may limit such analysis, as may the low representativeness of in situ observations, which greatly depend on instrument location and spatial density of the network (Haylock et al., 2008). Indeed, the daily high-resolution gridded datasets have a number of potential inaccuracies and errors. Generally, interpolation errors increase as the network density decreases, especially for variables with non-Gaussian spatial characteristics (e.g. precipitation), and degrade in areas of complex terrain (e.g. mountain areas, Hofstra et al., 2009).

Nevertheless, daily high-resolution gridded datasets derived from meteorological station measurements have become increasingly popular for regional scenario development since these observed datasets provide temporally complete 
time series of areal averages (and not point values) which can be directly compared to validate regional models (see, e.g. Frei et al., 2003; Suklitsch et al., 2011) or to calibrate the statistical downscaling methods (see, e.g. Schmidli et al., 2006, 2007).

Rigorous evaluation of distribution and frequency of extreme events is thus an important step in assessing the reliability of the datasets for climate downscaling and climate impact studies in this region. Indeed, developing regional gridded datasets is a key problem for climate change impact/adaptation studies and has become a strategic topic in national and international climate programmes, such as the ARCIS initiative (http://www.arcis.it/), and the EURO4M project (http://www.euro4m.eu/). ARCIS (the climatological archive for northern Italy) is a project run by the regional and provincial agencies for environmental protection in northern Italy with the ultimate aim of building a database of daily climatological data for northern Italy. The objective of the EU-funded EURO4M project (European Reanalysis and Observations for Monitoring) is to provide reliable information on the state and evolution of the European climate developing high-resolution datasets. Both projects are likely to make these datasets available in the next few months.

Taking into account these considerations, in this study we compare three public high-resolution datasets of interpolated precipitation: (i) EOBS (Haylock et al., 2008), (ii) MAP (Frei and Schär, 1998), and (iii) the recently developed NWIOI dataset (Ronchi et al., 2008; AAVV, 2011) over the Greater Alpine Region (GAR) and a subregion in northwest Italy (NWI). The EOBS dataset (Haylock et al., 2008) is the first publicly available daily gridded dataset covering the whole of Europe at a very high spatial resolution $(25 \mathrm{~km})$ and is continuously updated, making it one of the most widely used datasets and a most useful tool for the scientific community. Unfortunately, a number of studies have shown a potential weakness of this dataset in regions of sparse data (see, e.g. Kyselý and Plavcová, 2010; Herrera et al., 2012; Maraun et al., 2012, for the Czech Republic, Spain and UK, respectively).

Hofstra et al. (2009) compare MAP and EOBS (first version) over the GAR domain, finding a general underestimation of EOBS, larger errors in the Alps, and an overestimation of extreme precipitation. These results have been confirmed by Flaounas et al. (2012), who estimate the uncertainties of the EOBS dataset for temperature and precipitation over three domains covered by HyMeX (HYdrological cycle in the Mediterranean EXperiment, http://www.hymex.org/) stations: (i) Israel, (ii) coastal region of southern France, and (iii) northeastern Italy. Their analysis indicates that the EOBS uncertainties are rather important and cannot be neglected during development of the regional scenarios.

Here we extend these studies, (i) analyzing the latest available version of EOBS (v. 7.0), (ii) testing the new NWIOI dataset and (iii) calculating, in addition to standard measures of temporal similarity, their spatial similarity of aggregated values of a subset of the standard "moderate" extreme precipitation indices defined by the World Meteorological Organization (WMO) CC1/CLIVAR/JCOMM Expert Team on Climate Change Detection and Indices (ETCCDI; for more details see http://cccma.seos.uvic.ca/ETCCDI or WMO, 2009).

Specifically, EOBS v. 7.0 has approximately doubled the station density in the GAR domain with respect to the first EOBS version (passing from around 350 to 750 stations) through the inclusion of several records from northern Italy. A careful analysis of these series has revealed that most of the EOBS gridpoint series in northeastern Italy have to be shifted back by 1 day to have the maximum overlap of the measurement period. This result highlights the importance of assessing the consistency of new gridded observation versions that are developed when new historical instrumental data become available (through digitalization and data quality control).

Although no perfect gridded observations exist, some are more reliable than others - for example, through the inclusion of more observations. The new NWIOI covering the NWI domain is the dataset with the longest period with hundreds of stations over this region $(\sim 200)$. This grid allows a third term of comparison to be added, leading to greater reliability in the results of this assessment.

Finally, in the context of the development and evaluation of models for regional climate studies, of paramount importance is the assessment of uncertainties, not only of the models themselves but also of the validating datasets. Our analysis of day-count precipitation indices in the Alpine region should provide an easy, straightforward way for those who validate models to understand whether or not their results are within the gridded observation uncertainties. Indeed, the use of the standard ETCCDI indices makes comparative data analysis possible.

This paper is organized as follows: after this Introduction, Sect. 2 describes the observational datasets and the comparison measures used in this study, then Sect. 3 analyses the results, while Sects. 4 and 5 discuss and summarize the main conclusions of this study.

\section{Data and methods}

\subsection{Observations}

We consider three public high-resolution datasets of interpolated observations: (i) MAP (Frei and Schär, 1998), (ii) EOBS (Haylock et al., 2008), and (iii) NWIOI (Ronchi et al., 2008; AAVV, 2011). These datasets are daily observational grids for precipitation and are all produced using data from quality-controlled stations. Table 1 summarizes some details on these datasets.

We consider two domains (Fig. 1): (i) the Great Alpine Region (GAR; $2.25-17.25^{\circ} \mathrm{E}, 42.25-48.75^{\circ} \mathrm{N}$ ) and (ii) a zoom on the GAR, in northwest Italy (NWI; $6.50-9.50^{\circ} \mathrm{E}$, $\left.44.00-46.50^{\circ} \mathrm{N}\right)$. The first domain, GAR, covers the 
Table 1. The three datasets considered in this study showing spatial resolution, available period, number of stations over the GAR/NWI areas and links to their respective web pages.

\begin{tabular}{llllll}
\hline Dataset & Resolution & Period & GAR & NWI & Link \\
\cline { 4 - 5 } & & & \multicolumn{2}{c}{ (no. of stations) } & \\
\hline MAP v. 4.0 & $\begin{array}{l}0.25^{\circ} \times 0.1667^{\circ} \\
(\sim 25 \mathrm{~km} \times 20 \mathrm{~km})\end{array}$ & $1971-1998$ & $\sim 6500$ & $\sim 60$ & http://www.map.meteoswiss.ch/map-doc/rr_clim.htm \\
EOBS v. 7.0 & $\begin{array}{l}0.25^{\circ} \times 0.25^{\circ} \\
(\sim 25 \mathrm{~km} \times 25 \mathrm{~km})\end{array}$ & $1950-2013$ & $\sim 750$ & $\sim 10$ & http://eca.knmi.nl/ \\
NWIOI v. 2.0 & $\begin{array}{l}0.125^{\circ} \times 0.125^{\circ} \\
(\sim 15 \mathrm{~km} \times 15 \mathrm{~km})\end{array}$ & $1958-2013$ & $\begin{array}{l}\text { Available only } \\
\text { for NWI }\end{array}$ & $\sim 200$ & http://rsaonline.arpa.piemonte.it/meteoclima50/ \\
\hline
\end{tabular}
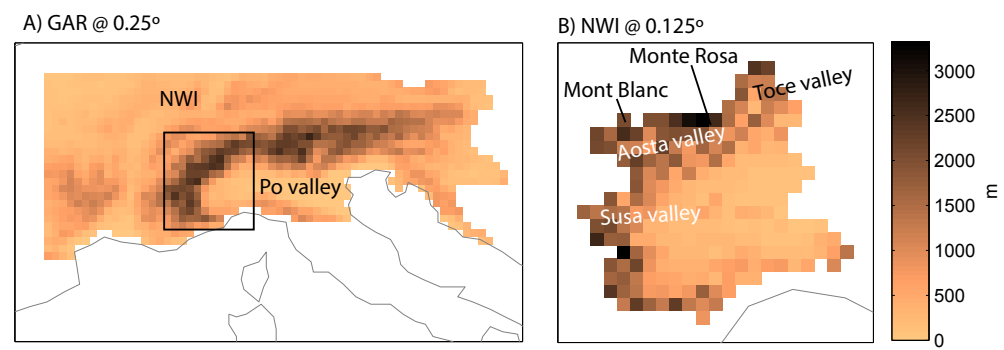

Fig. 1. (A) Topography of GAR domain as represented by EOBS at $0.25^{\circ} \times 0.25^{\circ}$. The inset shows the NWI domain. (B) Topography of NWI domain as represented by NWIOI at $0.125^{\circ} \times 0.125^{\circ}$. The pictures report the geographic locations mentioned in the text.

European Alps and the adjacent plains, while NWI covers the ridge of the Alps of the Piedmont and Aosta Valley regions (which have the highest peaks of the Alps, including Mont Blanc, $4810 \mathrm{~m}$ ) and the adjacent plains.

The MAP dataset, composed of $\sim 6500$ stations, covers the GAR area and provides reliable data only over the period 1971-1990. For more recent years (1991-1998), the station coverage for northern Italy is lower and many of the Italian stations are suspected of having data quality problems (Frei and Schär, 1998; Hofstra et al., 2009). MAP was developed using a modified version of the SYMAP interpolation method of Shepard (1984), which consists in areal-averaged values weighing the bias-uncorrected, quality-controlled observations in a search radius around the gridpoint. This dataset is considered reliable for reproducing the mesoscale patterns of the present alpine climatology, at least qualitatively (Frei and Schär, 1998).

EOBS is the state-of-the-art publicly available highresolution daily dataset for Europe, based on a network of more than two thousand stations. Compared to MAP, EOBS is based on a lower number of stations - around 750 records over the GAR domain. This grid was derived applying the kriging method in a three-step process. First, monthly totals were interpolated using thin plate splines taking into account latitude, longitude and elevation. It should be noted that, as we discuss in Sect. 3.3, taking into account the elevation in the interpolation process could be a key factor in explaining the larger discrepancy from MAP and NWIOI in the spatial patterns of the ETCCDI values. Second, daily anomalies (i.e. departures from the monthly totals) were interpolated using an indicator kriging and, third, the magnitudes were interpolated using universal kriging for the occurrence outcomes (Haylock et al., 2008).

NWIOI covers the NWI domain and is the dataset with the longest period covered by hundreds of daily stations over this region $(\sim 200)$. The interpolation process is based on the optimal interpolation (OI) technique. The role of this algorithm in atmospheric modelling is extensively described by Kalnay (2003). The method used to generate NWIOI is an implementation of OI, described in detail by Uboldi et al. (2008) and concisely presented here. This method differs from kriging since it assumes a meteorological background as first-guess field. For NWIOI, a "pseudo" background field is built from the data in order to represent the main spatial trends present in the field and detected by the observations. This algorithm then produces a precipitation interpolation field through the background field corrected by a linear combination of the differences between this first guess and the rain station records. The coefficients of the linear combination are derived by minimizing the covariance matrices of observed and background field data. This method makes it possible to weigh the contribution to the precipitation value at each gridpoint from the nearest observation data, while using an external background field makes it possible to achieve time homogeneity and spatial coherence in the final dataset. 
To compare the three datasets, we focus on the common reliable period, 1971-1990, and the daily outputs of the MAP and NWIOI are bilinearly interpolated (upscaled) from their original resolution to the grid defined by EOBS. While this process may introduce a small additional uncertainty, we considered it negligible.

\subsection{Comparison measures}

Two main approaches have been applied to compare the three datasets (see Murphy, 1993, for a description of verification methods). Firstly, as a measure of the temporal agreement between the time series, the datasets are compared at a gridpoint basis using standard verification measures (e.g. correlation). Specifically, at each gridpoint, we compute the relative mean absolute error (MAEr) relative to the observed mean of the reference dataset (in order to remove the influence of the precipitation amount) and the Pearson correlation (CORR; we calculate both the Pearson and the Spearman correlation without qualitative differences) between the datasets. We establish as reference datasets the MAP dataset for the GAR domain and NWIOI for the NWI domain since it is reasonable to expect smaller errors for those grids with higher station density. The spatial median and interquartile range (IQR) of the scores considered are then reported above the relative figures (Figs. 2 and 3).

Secondly, we consider standard verification measures (e.g. bias) to assess the spatial agreement of annually or seasonally aggregated values between the datasets. Specifically, we compare the observed climatologies (spatial patterns) of a subset of the standard ETCCDI indices (WMO, 2009). Table 2 describes the ETCCDI indices that we calculate (total precipitation amount, number of precipitation days with precipitation greater than $1 \mathrm{~mm}, 10 \mathrm{~mm}$, or $20 \mathrm{~mm}$, maximum precipitation over 1 and 5 days and the number of consecutive wet or dry days). All the indices are calculated on annual and seasonal scales over the common period 1971-1990 (except those which make no sense on a seasonal scale, such as the CDD index since the longest period without rainfall can occur across two seasons).

A graphic summary of how closely the spatial patterns of the different datasets match each other is provided by the Taylor diagram (Taylor, 2001). The Taylor diagram makes it possible to summarize the similarity between two spatial fields in a single bidimensional plot in order to reproduce (i) the spatial characteristics (spatial correlation, $C$ ), (ii) the spatial variability (standard deviation, $S$ ), and (iii) the quantities (centred root-mean-square error, $R$ ). It should be noted that the statistics have been normalized by dividing both the centred root-mean-square and the standard deviations by the standard deviation of the reference datasets. In this way it is possible to compare the different indices. Furthermore, in order to include information about overall biases, the colour of each point indicates the difference between the simulated and observed mean, normalized by the mean of the reference grid (bias or mean error, $M$ ). The reference datasets are indicated in the plot as OBS at $(1,0)$ on the axis. Thus, more similar datasets to the reference datasets are closer to this point.

\section{Results}

\subsection{Temporal similarity}

In this section we estimate the temporal similarity of the three datasets. To this aim, for each gridpoint we computed the MAEr and the CORR between a dataset and the "reference" ones (MAP for the GAR domain and NWIOI for the NWI domain, as explained in the previous section). Figure 2 shows the spatial distribution of the accuracy metrics over the GAR domain. Both metrics highlight that although agreement in the GAR domain is generally quite high (i.e. correlation with average values around $90 \%$ ), there are larger differences in northeastern Italy (Figs. 2a and b).

Starting from these results, we scrutinized the data and found a "shifting error" in the data assignment of the daily times series of the EOBS datasets in this area. During the period under study (1971-1990), there was an important change in the measurement practice in Italy. Until 1986, the daily reading time was at 09:00 UTC and the "Servizio Idrografico Mareografico Nazionale" (the body specifically responsible for collecting the data in Italy) assigned the daily accumulated rainfall of a day $\mathrm{D}$ to the precipitation registered from 09:00 UTC on day D-1 to 09:00 UTC on day D. That is, these station records have to be shifted back by 1 day to have maximum overlap of the measurement period (09:00-09:00 UTC) and the 00:00-24:00 UTC time period. From 1987 on, the reading time was generally at 00:00 UTC and the data assignment started to be correct. We confirmed this shifting error directly by checking the assigned data in the original database for the stations provided to the EOBS developers (V. Pavan, personal communication, 2013). For each year in the period 1971-1990, we also calculated the cross correlation between each gridpoint time series of the MAP and EOBS dataset and plotted the lag where there was the maximum correlation between the time series. This analysis confirms that in northeastern Italy, the EOBS datasets display the highest correlation with MAP at +1 day's time lag during the period 1971-1986. The dotted black circles in Fig. $2 \mathrm{a}$ and $\mathrm{c}$ indicates the EOBS gridpoints whose accumulated precipitation series are erroneously shifted, while the highest correlation between MAP and EOBS is always at the time lag 0 for the other gridpoint of the domain, and over the whole GAR domain during the period 1987-1990, with some minor exceptions. In particular, for the period 1987-1990 we have not accurate metadata and the results of the cross-correlation analysis does not provide a guidance to clearly say if a series needs to be date shifted or not. Thus, we did not make changes to the dataset during these four years. Further analysis should be done to face this "date shift problem", when more data and metadata will be available. 
Table 2. Climatic mean and extreme indices for precipitation used in this work (see also ETCCDI, http://cccma.seos.uvic.ca/ETCCDI).

\begin{tabular}{llc}
\hline Label & Description & Units \\
\hline PRCPTOT & total precipitation & $\mathrm{mm}$ \\
R1 & number of days with precipitation over $1 \mathrm{~mm} \mathrm{day}^{-1}$ (i.e. rainy days) & day \\
SDII & mean precipitation amount on a wet day & $\mathrm{mm}$ \\
R10 & number of days with precipitation over $10 \mathrm{~mm} \mathrm{day}^{-1}$ & day \\
R20 & number of days with precipitation over $20 \mathrm{~mm}^{-1}$ & day \\
RX1DAY & maximum precipitation in 1 day & $\mathrm{mm}$ \\
RX5DAY & maximum precipitation in 5 days & $\mathrm{mm}$ \\
CWD & consecutive wet days $(>1 \mathrm{~mm})$ & day \\
CDD & consecutive dry days $(<1 \mathrm{~mm})$ & day \\
\hline
\end{tabular}
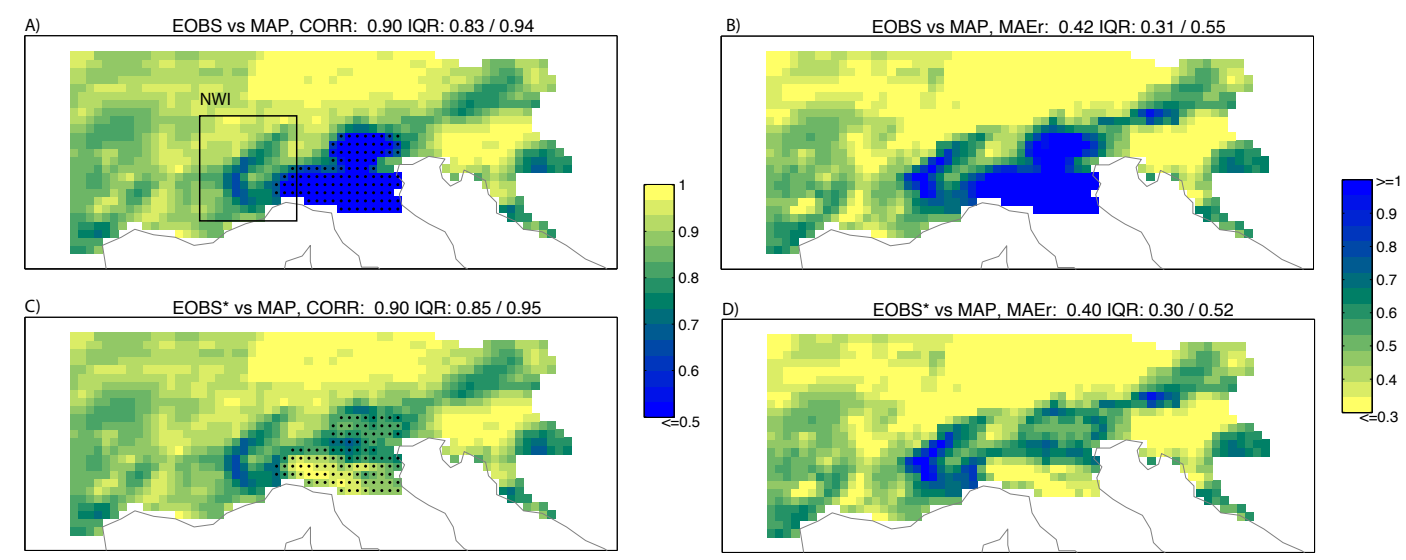

Fig. 2. (A) CORR (Pearson correlation) comparing EOBS and MAP over GAR; dotted black circles indicate the EOBS gridpoints whose accumulated precipitation series have to be shifted back by 1 day to have maximum overlap of the measurement period. The inset shows the NWI domain. (B) MAEr comparing EOBS and MAP. (C) and (D) are the same as (A) and (B) but for the shift-corrected EOBS (EOBS*). The values at the top of each map are the median and interquartile range (IQR) of the spatial distribution of the corresponding score.

We then repeated the analysis considering a shift-corrected version EOBS. The corrected EOBS version clearly outperforms the original (Fig. 2). Thus, in the following analysis we consider only the shift-corrected version of EOBS. Compared to the first version of EOBS analysed by Hofstra et al. (2009), the shift-corrected EOBS v. 7.0 provides a slightly better regionally averaged skill $(\mathrm{CORR}=0.90$ versus 0.88 , and MAEr $=0.40$ versus 0.51 , as reported in Table 2 of Hofstra et al., 2009) and better agreement over northeastern Italy, where the station density has increased significantly.

Generally, the greatest inconsistencies (with MAEr values even greater than $100 \%$ ) between the datasets are in the NWI region. Figure 3 concerns the analysis of this area. Now the corrected EOBS is considered. This figure indicates greater correspondence between MAP and NWIOI than between MAP or NWIOI and EOBS. As expected, a common pattern is the greater discrepancy between the grids over the mountainous areas. In particular, the greater differences between MAP and NWIOI on the Aosta Valley border could be related to a lack of stations outside the Italian border in the NWIOI dataset.
These results are valid even when considering the seasonal series (not shown) instead of the annual ones, with temporal agreement measures of the same order of magnitude (e.g. $C \simeq 90 \%$ and $\mathrm{MAEr} \simeq 45 \%$, except in JJA with $\mathrm{MAEr}=52 \%)$.

\subsection{Spatial similarity of aggregated indices over GAR}

The annual climatology (spatial pattern), averaged over the studied period (1971-1990) for the precipitation indices shown in Table 2, has been calculated for the three datasets (see Fig. 4 for the GAR domain and Fig. 5 for the NWI domain). The panels in Fig. 4 show the annual values of the indices for MAP (first column), EOBS (second column) and the differences of EOBS minus MAP (third column); the numbers below the figures indicate the calculated similarity scores (i.e. those used in the Taylor diagram).

This figure shows quite good spatial similarity between MAP and EOBS over the GAR domain, with spatial correlation generally around $80 \%$. The similarity between the two datasets is generally high for PRCPTOT, R1, SDII and R10, 

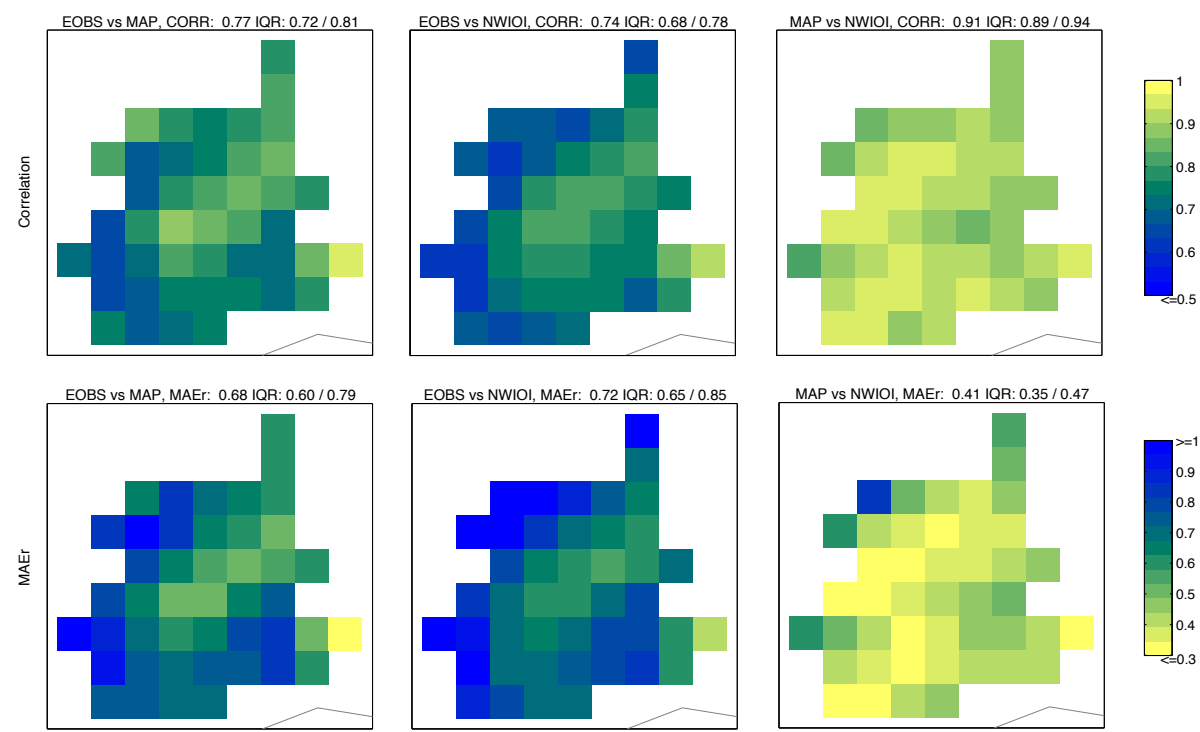

Fig. 3. CORR (top panels) and MAEr (bottom panels) comparing EOBS (shift corrected), MAP and NWIOI over NWI. The values at the top of each map are the median and the interquartile range (IQR) of the spatial distribution of the corresponding score.

with a mean error of less than $-10 \%$ (the minus sign indicates that EOBS has, on average, lower values than MAP), similar standard deviation, and a centred root-mean-square error of less than 0.6. The difference maps (third column) show that although generally EOBS has lower values than MAP, over the Alpine chain EOBS has higher values, especially over NWI. The following analysis over the NWI domain focuses on this area. For R20 and for the extremes indices RX1DAY and RX5DAY, the discrepancies are greater, with a mean error of between $-10 \%$ and $-20 \%$. Furthermore, the three areas with the highest maximum values, the south of France, the Toce Valley (in the north of Piedmont) and the northeast of Italy, are not highlighted by EOBS. Finally, the frequency indices CWD and CDD, indicate a quite high spatial similarity.

As well as the dataset assessment, Fig. 4 also provides useful information on the climatology of the ETCCDI indices over the GAR domain. Here, despite the relatively small size of the selected region (around $1200 \mathrm{~km} \times 700 \mathrm{~km}$ ), the spatial distributions of daily precipitation indices vary widely. Topography, distance from the Mediterranean and position play a dominant role in determining the spatial distribution of these indices. On this scale, total precipitation is quite well correlated with the alpine chain (despite several exceptions on a smaller scale), ranging from around $1000 \mathrm{~mm} \mathrm{yr}^{-1}$ over the lowlands to maximum values of over $2000 \mathrm{~mm} \mathrm{yr}^{-1}$ over the mountains.

The climatology map of the R1 indicates quite a strong south to north gradient, with more than 120 days of rain (i.e. once every 3 days, on average) in the northern part of the GAR domain. In this area the precipitation intensity (SDII index) is quite low, while the maximum values, amounting to over $15 \mathrm{mmday}^{-1}$, are located in the south of France, the Toce Valley and the northeast of Italy. Over these three areas and the reliefs, there are more than 60 days with precipitation greater than $10 \mathrm{mmday}^{-1}$ and 30 days with precipitation greater than $20 \mathrm{~mm}_{\text {day }}{ }^{-1}$. These indices, R10 and R20, are spatially similar to the PRCPTOT index, whereas the extremes indices are more similar to the climatology map of SDII, where the three areas mentioned above have RX1DAY values over $140 \mathrm{mmday}^{-1}$ and RX5DAY values over $250 \mathrm{~mm}(5 \text { days })^{-1}$. Finally, the frequency indices CWD and CDD again confirm the great spatial variability of the GAR domain, from regions with more than 15 consecutive days of rain (in the north) to regions with 40 consecutive dry days (in the Po Valley).

\subsection{Spatial similarity of aggregated indices over NWI}

Figure 5 shows the climatology (averaged over the period 1971-1990) for the precipitation indices given in Table 2 for the three datasets. The panels in Fig. 5 indicate the annual values of the indices for MAP (first column), EOBS (second column), NWIOI (third column; the grid taken as reference) and their respective differences (fourth and fifth columns). Table 3 summarizes the spatial similarity scores of these maps.

This comparison shows the high agreement between MAP and NWIOI (e.g. spatial correlation around $70-90 \%$, depending on the index). This is an important result since it builds confidence in using the MAP and NWIOI datasets for climate studies. Indeed, the former reference dataset is commonly accepted as a reliable description of the mesoscale variability of precipitation in the Alps (Frei and Schär, 1998). The latter dataset, based on a high number of stations (around 
Table 3. Spatial validation scores (mean error $M$, standard deviation $S$, centred root mean square $R$, correlation $C$ ) for the MAP and EOBS values, with regard to the NWIOI values for the climatologies of the annual precipitation extremes indices shown in Table 2 over Piedmont and the Aosta Valley.

\begin{tabular}{lllllllll}
\hline Index & \multicolumn{9}{c}{ MAP } & \multicolumn{7}{c}{ EOBS } \\
\hline PRCPTOT & $M=0.01$ & $S=1.03$ & $R=0.51$ & $C=0.87$ & $M=-0.05$ & $S=1.10$ & $R=1.45$ & $C=0.04$ \\
R1 & $M=-0.05$ & $S=1.35$ & $R=0.77$ & $C=0.83$ & $M=-0.06$ & $S=1.51$ & $R=1.15$ & $C=0.65$ \\
SDII & $M=0.07$ & $S=0.97$ & $R=0.39$ & $C=0.92$ & $M=0.01$ & $S=0.81$ & $R=1.54$ & $C=-0.43$ \\
R10 & $M=0.02$ & $S=0.93$ & $R=0.59$ & $C=0.82$ & $M=0.08$ & $S=1.47$ & $R=1.92$ & $C=-0.18$ \\
R20 & $M=0.06$ & $S=0.95$ & $R=0.44$ & $C=0.90$ & $M=-0.08$ & $S=1.04$ & $R=1.64$ & $C=-0.29$ \\
RX1DAY & $M=0.05$ & $S=1.10$ & $R=0.49$ & $C=0.90$ & $M=-0.18$ & $S=0.53$ & $R=1.13$ & $C=-0.00$ \\
RX5DAY & $M=0.01$ & $S=0.97$ & $R=0.40$ & $C=0.92$ & $M=-0.23$ & $S=0.37$ & $R=1.16$ & $C=-0.28$ \\
CWD & $M=-0.13$ & $S=0.79$ & $R=0.69$ & $C=0.72$ & $M=-0.16$ & $S=0.68$ & $R=1.21$ & $C=0.01$ \\
CDD & $M=-0.02$ & $S=1.20$ & $R=0.58$ & $C=0.87$ & $M=-0.00$ & $S=1.76$ & $R=1.04$ & $C=0.86$ \\
\hline
\end{tabular}

200), shows high agreement with MAP, suggesting the reliability of these two grids. Instead, the low or even negative spatial correlations for the EOBS grid suggest that this dataset, at least for certain studies such as the analysis of the regional alpine climatology, cannot be considered reliable.

We underline that this conclusion is supported by the evidence that its spatial pattern, which is very different from those of MAP and NWIOI over the NWI region, is implausible also from a physical point of view. The spatial variability of the daily precipitation indices is very high over the NWI domain, in spite of its relatively small surface area (around $29000 \mathrm{~km}^{2}$ ). The maximum values (up to $2000 \mathrm{~mm} \mathrm{yr}^{-1}$ ) are in the northeast, close to the foothills adjacent to the Monte Rosa chain. The position of this alpine chain, intercepting the humid winds from the south, and its relative proximity to the sea, are the factors that explain these high averaged values. The lower values are in the Po Valley, about $800 \mathrm{~mm} \mathrm{yr}^{-1}$, and in some inner valleys, like for example in the Aosta Valley (in the north) and the Susa Valley (in the west), which are sheltered from the humid flows to the blocking action of mountain ridges. On the contrary, the EOBS correction with altitude shifts the precipitation peaks from the pre-mountain range to the mountains located at the border of the NWI domain. It should be noted that while for MAP and NWIOI there is no significant linear relationship between orography and mean precipitation, the correlation for EOBS is around $88 \%$ ( $78 \%$ considering the mean daily variability). Please also notice that, while in alpine areas it is generally recognized that the stations tend to be at low elevations (e.g. mountain valleys), correct compensation of the measurement error deriving from this incomplete representative clustering is not a trivial question. This is mainly due to the spatial variability of the non-linear precipitation-elevation relationships.

Considering the spatial patterns of the ETCCDI indices, the rainy days index also shows high spatial variability (from around 80 up to 160 days $\mathrm{yr}^{-1}$ ), while the intensity of precipitation varies less, with averaged values of $9 \mathrm{mmday}^{-1}$. The indices R10 (average around 30 days $^{-1}$ ) and $R 20$ (average around 12 days $\mathrm{yr}^{-1}$ ) are spatially similar to the
PRCPTOT index. The extremes index RX1DAY (RX5DAY) shows the higher spatial variability, with values ranging from $40 \mathrm{~mm} \mathrm{day}^{-1}\left(100 \mathrm{~mm}(5 \text { days })^{-1}\right)$ over the lowlands and inner valleys up to $120 \mathrm{~mm} \mathrm{day}^{-1}\left(250 \mathrm{~mm}(5 \text { days })^{-1}\right)$ over the northwest alpine area. Finally, the CWD index shows the highest difference between the MAP and NWIOI datasets $(M=-13 \%)$. The regional average of the CWD index varies between about 8/9 days with continuous rain. The regional averaged period with consecutive dry days (CDD index) is around 30 days $y^{-1}$.

Focusing on the seasonal scale, the spatial similarity of the MAP and EOBS datasets in reproducing the NWIOI climatologies of the ETCCDI indices is shown in the Taylor diagrams displayed in Fig. 6. For all the seasons, EOBS shows greater discrepancies, especially in winter, probably linked to snow measurement problems.

The precipitation in the NWI domain is characterized by large variability: not only spatially, but also in time. In Fig. 7 we compare the three datasets on a monthly scale in order to assess their correspondence for annual cycles of certain indices. We consider the annual cycle of the indices PRCPTOT, R1, R20, and RX1DAY, averaging the gridpoint indices in the domain and considering the common period 1971-1990. In this case, too, there is greater agreement between NWIOI and MAP, while EOBS underestimates the peaks of the annual cycle, particularly the extremes index RX1DAY.

\section{Discussion}

In the context of climate studies, the analysis of highresolution daily observation is of the utmost importance. These datasets allow the main climatic features of their area of reference to be defined and can, for instance, be used to analyse the indices of daily precipitation extremes. Furthermore, gridded datasets derived from meteorological station measurements are most important in setting up the basis for the development of regional climate scenarios. Unfortunately, a number of constraints involving climate data 

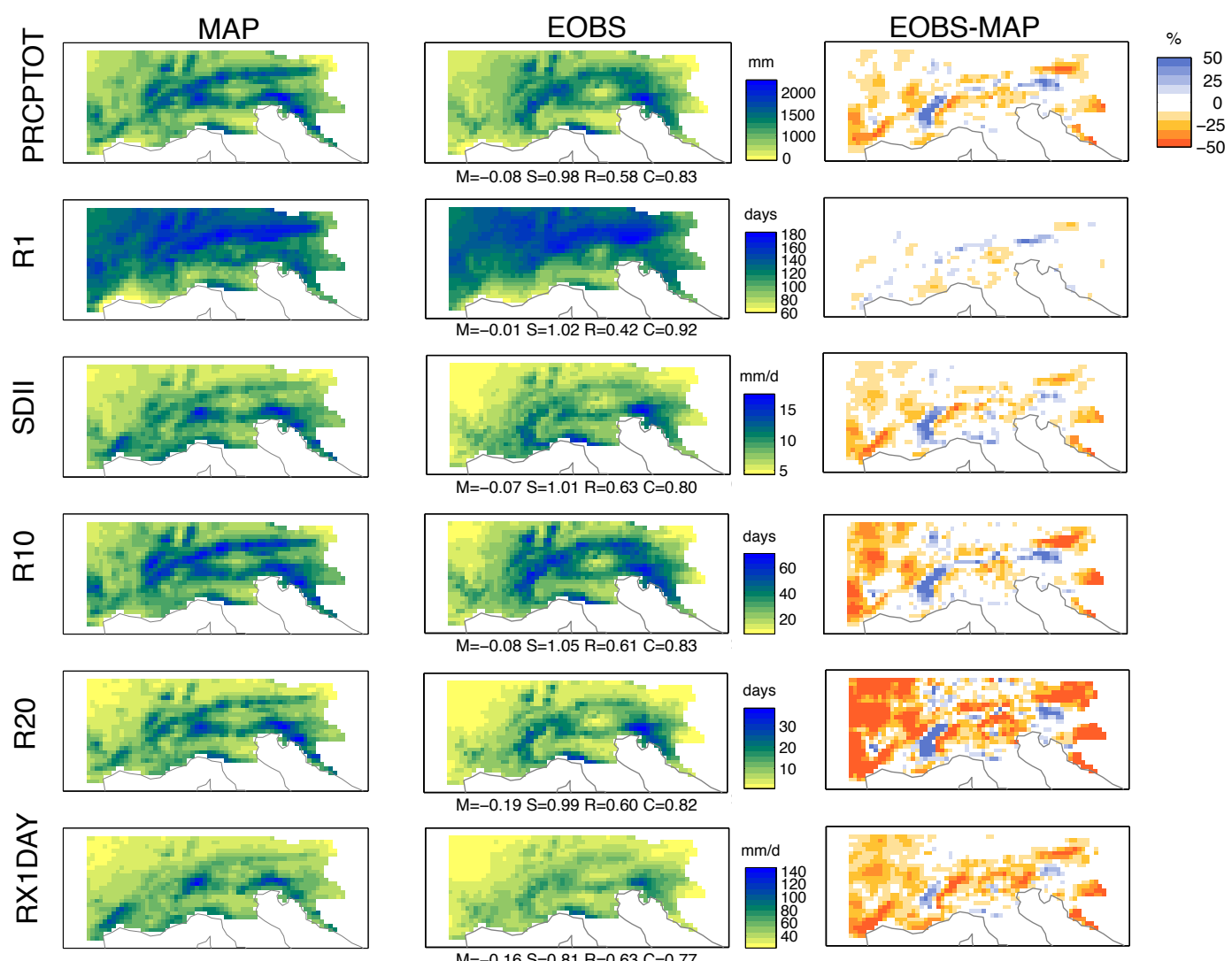

$M=-0.16 \mathrm{~S}=0.81 \mathrm{R}=0.63 \mathrm{C}=0.77$
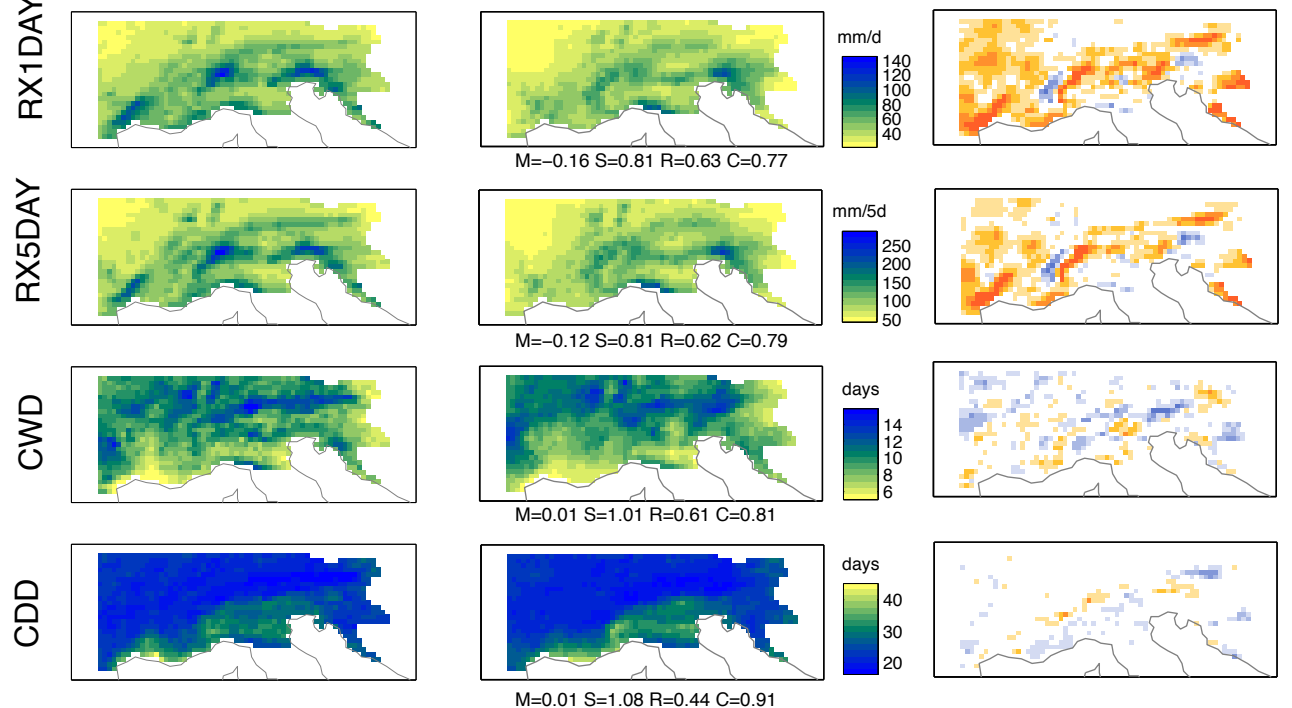

Fig. 4. Spatial distribution of mean values (averaged over the period 1971-1990) of the MAP (left), EOBS (central) and their differences (right), for the annual precipitation extremes indices shown in Table 2. The spatial validation scores (mean error $M$, standard deviation $S$, centred root mean square $R$, correlation $C$ ) for the EOBS values, with respect to the MAP values, are given below the corresponding panels.

(i.e. availability of the data and their quality) could limit the analysis of daily fields, which is even more challenging in the alpine area, given the complexity of the topography in this region.

These reasons have motivated this study, in which we have compared three public datasets over the Alpine area: (i) MAP, (ii) EOBS, and (iii) NWIOI. We have compared these datasets over two domains (GAR and NWI), investigating their temporal similarity by means of certain standard descriptive statistics (CORR and MAEr), and their spatial si- milarity, comparing the spatial climatologies of several ETCCDI extreme precipitation indices on an annual and seasonal scale.

Each dataset has limitations that need to be considered. The MAP grid, covering the Great Alpine Area, is based on thousands $(\sim 6500)$ of quality-controlled stations, but is available (and reliable) only for the period 1971-1990. It would be really desirable for it to be kept updated. The EOBS grid, covering the whole of Europe, is based on fewer stations over the GAR domain, around only 750. Its advantage is its 

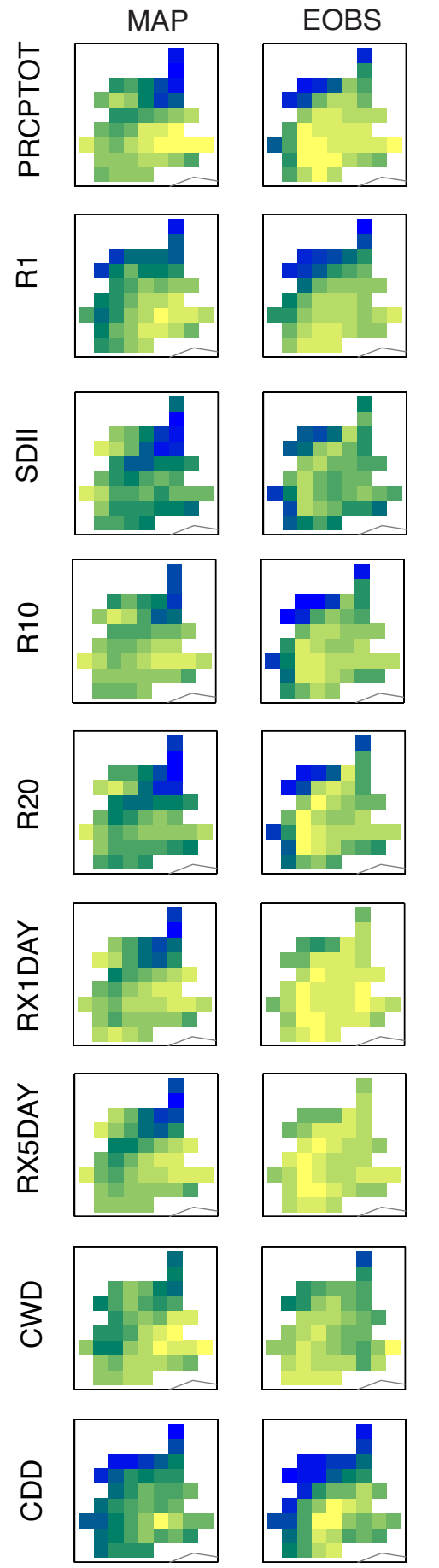
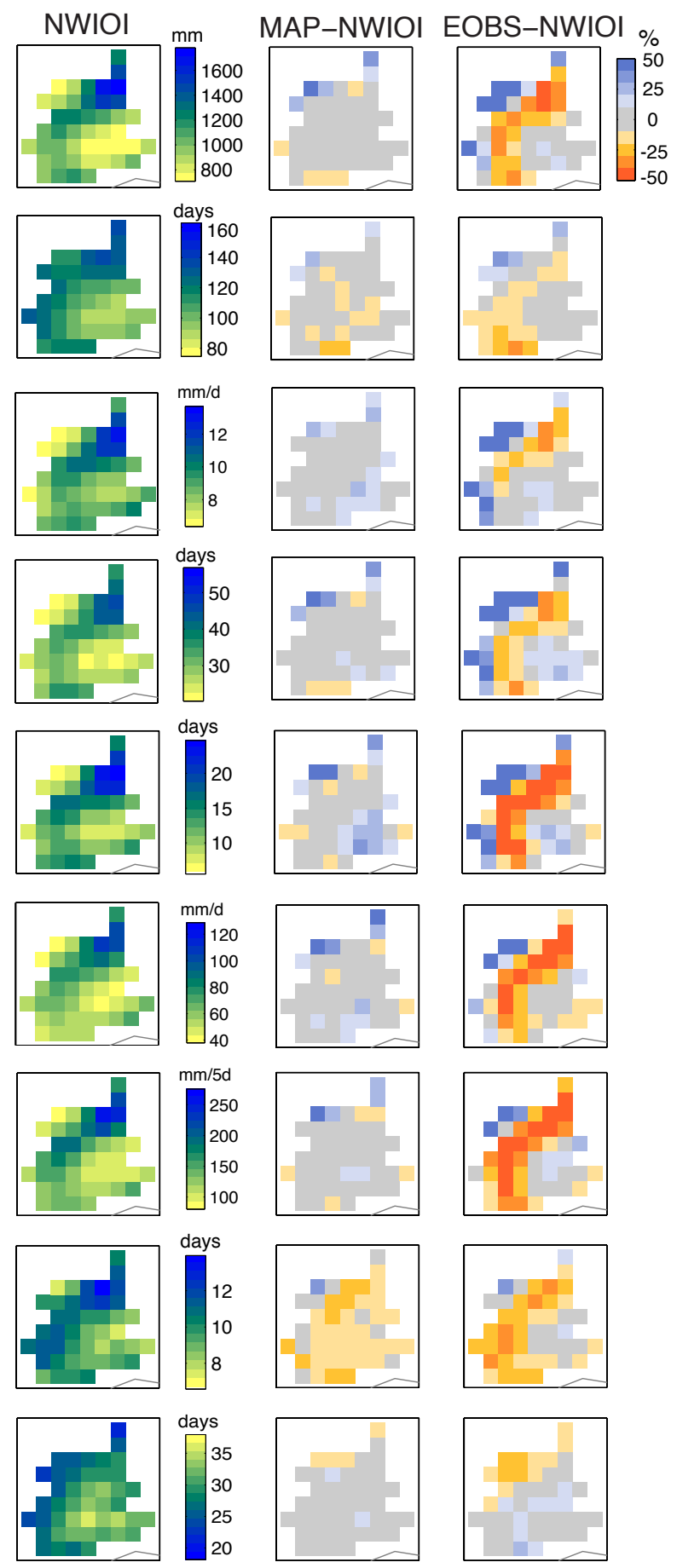

Fig. 5. Spatial distribution of mean values (averaged over the period 1971-1990) of the MAP, EOBS and NWIOI, and their respective differences, for the annual precipitation extremes indices shown in Table 2 over Piedmont and the Aosta Valley.

continuous updating. Finally, the NWIOI, based on hundreds of stations, has a very high spatial resolution and is continuously updated. However, it covers only a part of the GAR domain (NWI).

Given these considerations, EOBS could be very attractive to users who need constantly updated high-resolution data or data that simply cover recent periods over the GAR domain. The numerous studies that use this data to analyse past climatology or to evaluate the regional scenarios (by means of RCMs or statistical downscaling methods) confirm this fact. However, this grid in particular may suffer from a number of potential uncertainties (Haylock et al., 2008), since in general 
DJF

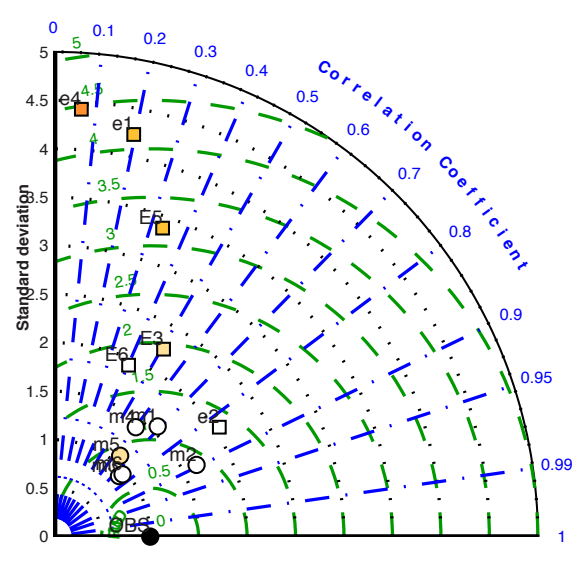

JJA

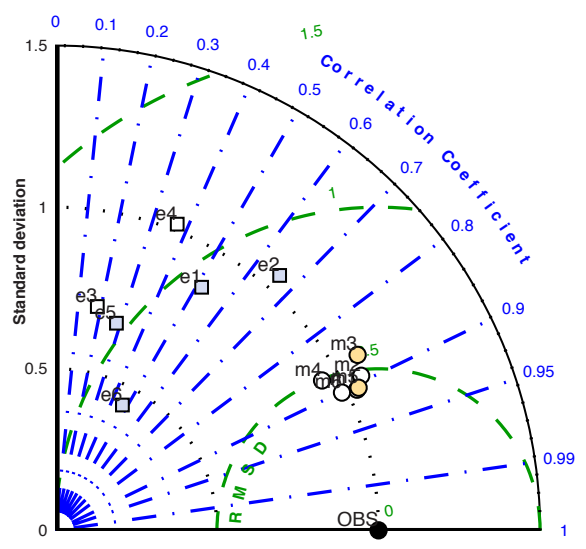

MAM

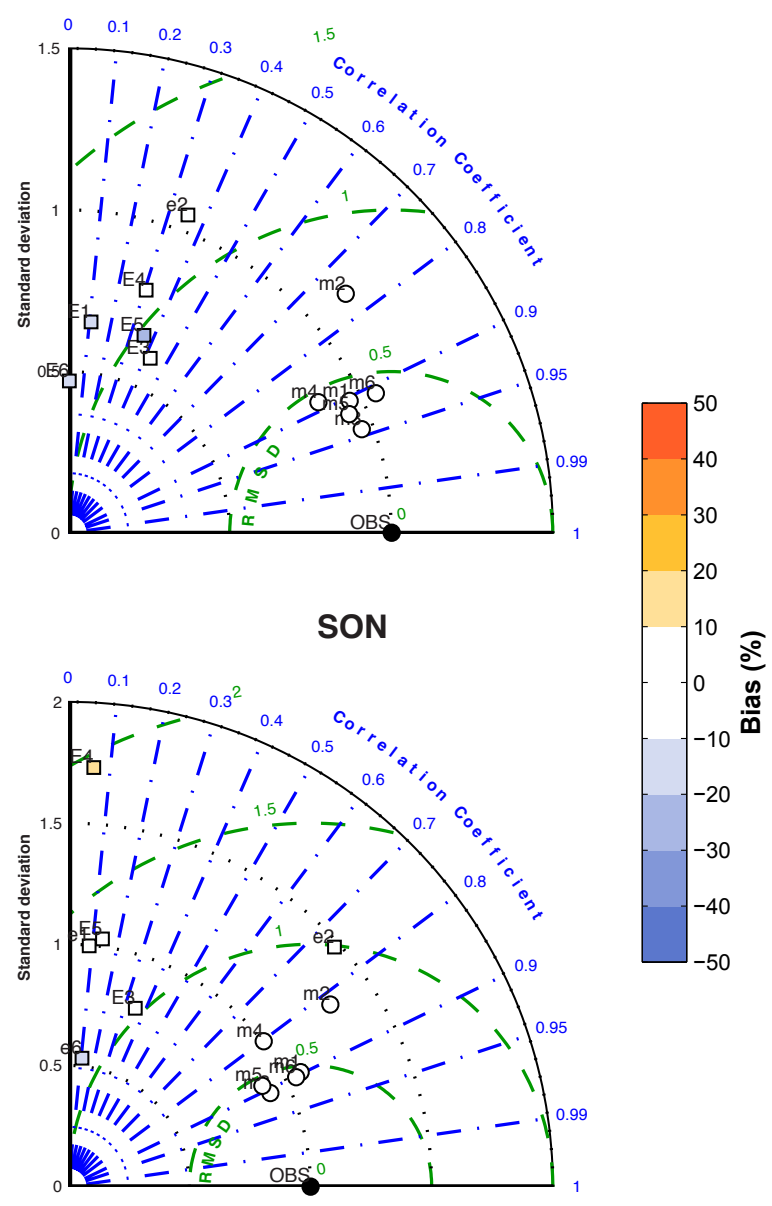

Fig. 6. Taylor diagrams for seasonal precipitation climatology over the NWI domain. The most similar points to the NWIOI dataset are closer to the point indicated as OBS. The squares with a small $e$ are used for EOBS (a capital $E$ is used for negative correlations), while the circles with the letter $m$ are for MAP. The colours indicate the bias (in percentage with respect to the NWIOI value) of the dataset. The numbers correspond to the different indices: $1=$ PRCPTOT; $2=\mathrm{R} 1 ; 3=\mathrm{SDII} ; 4=\mathrm{R} 10 ; 5=\mathrm{R} 20 ; 6=\mathrm{RX} 1 \mathrm{DAY}$.

the accuracy decreases as the network density decreases. Our study highlights that this dataset may be considered reliable only for mean values over the GAR domain and could indicate, even for a relatively small area like NWI region, whether a year is above or below normal. Indeed, although the temporal correlation of the daily series in this region is approximately $70 \%$ (see Sect. 3.1), the EOBS grid seems unable to reproduce the spatial precipitation variability over this region. That is, EOBS could indicate whether a year is above or below normal over the NWI domain, while across this domain it might not highlight which places are wet or dry. It should be noted that Ciccarelli et al. (2008) show that the interannual precipitation variability over NWI is generally coherent over the entire area; that is, dry (wet) years are usually dry (wet) over the whole region. This means that a few stations (such as those that compose the EOBS grid) allow the interannual variability of the precipitation to be captured, at least to some extent. However, the high biases for the extremes indices (e.g. Fig. 5) suggest that EOBS might only indicate whether a year is above or below normal, while it cannot provide reliable quantitative information.

Finally, it should be noted that these datasets may be affected by common measurement errors, such as undercatch due to wind or evaporation losses and/or snow drift into the rain gauge. The resulting bias could be an averaged underestimation of around $10 \%$ (Frei and Schär, 1998). However, none of the three datasets takes into account a correction term for these errors. So possible systematic underestimation of the amount of precipitation should therefore be taken into account.

\section{Summary and conclusions}

Our assessment of the Alpine region, focusing on temporal similarity, indicates that (i) most of the EOBS gridpoint series in northeastern Italy have to be shifted back by 1 day to 

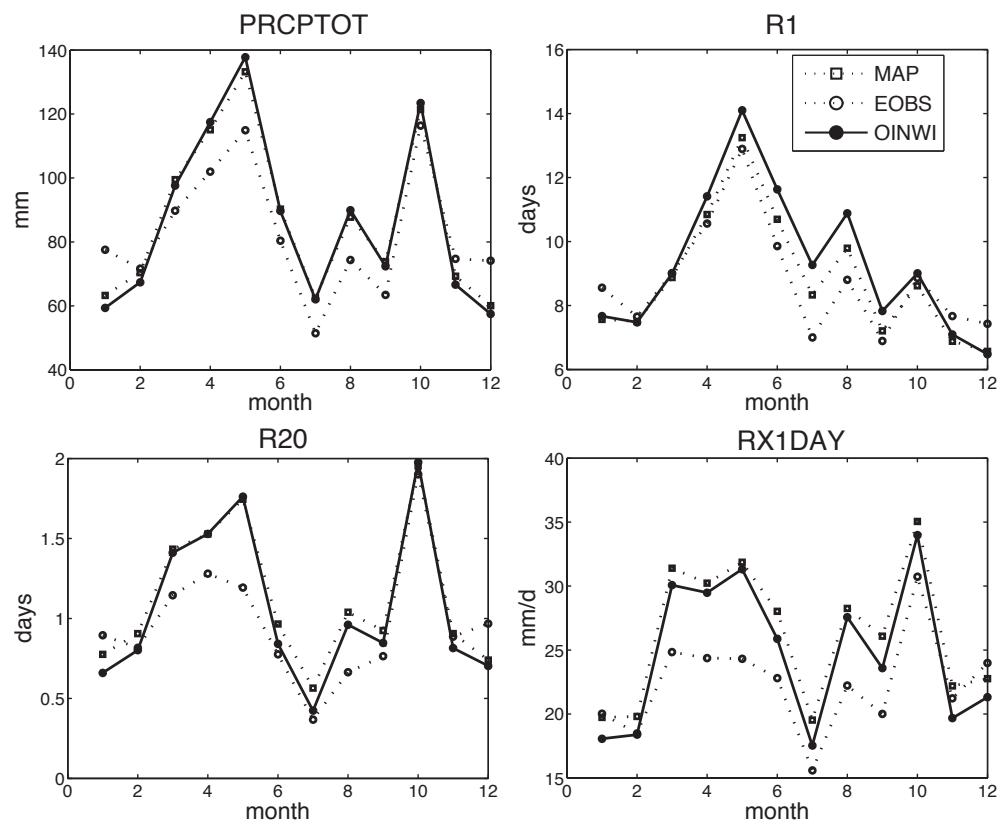

Fig. 7. Seasonal cycle (averaged monthly values) of spatially averaged indices. Solid circles and a continuous line represent the NWIOI data, empty squares and a dashed line represent the MAP data, while empty circles and a dashed line represent the EOBS data.

have maximum overlap of the measurement period, (ii) the shift-corrected EOBS version generally shows good agreement in terms of temporal correlation with MAP on the GAR domain, and (iii) there is also a relatively good temporal agreement between the three datasets over the NWI domain, with higher similarity between NWIOI and MAP than between MAP or NWIOI and EOBS.

Focusing on spatial similarity of ETCCDI climatologies, the main results are the following: (i) highest discrepancies have been found for the extremes indices, both on an annual and seasonal time scale (the highest differences are in winter); (ii) EOBS cannot be used for spatial climatology of extreme indices over the GAR domain and for any index over NWI (the spatial correlations with NWIOI are even negative and show unrealistic precipitation patterns over the Alpine chain); (iii) EOBS generally has lower values than MAP in most of the GAR domain, probably because it is based on fewer stations and the resulting interpolated fields are oversmoothed (Hofstra et al., 2010).

MAP and NWIOI generally show good agreement, which contributes to a greater confidence in using these databases. The main practical limitations of these datasets are that MAP covers only $20 \mathrm{yr}$, while NWI covers a relatively small region. Unfortunately, EOBS, which is continually updated and covers the entire domain, has shown the greatest discrepancies.

The users should carefully take into account the limitations of the observed datasets. Without proper high-resolution observations it is difficult or impossible to develop reliable high-resolution scenarios. Thus, once again, this work em- phasizes the importance of making climate data accessible for research activities.

Our future research would address the problem of the impact of climate change on hydrology, evaluating and calibrating the high-resolution outputs of COSMO-CLM (Zollo et al., 2012) by means of a hybrid downscaling strategy (Turco et al., 2011). This study is the first step in this direction, providing the groundwork to choose the most reliable dataset to develop regional scenarios over the Alpine region.

Acknowledgements. We acknowledge the EOBS dataset from the EU-FP6 project ENSEMBLES (http://ensembles-eu. metoffice.com) and the data providers in the ECA\&D project (http://eca.knmi.nl). We thank C. Frei for the MAP dataset. This work was funded by the Projects "GEMINA" and "NextData" of the Italian Ministry for Education, University and Research, and the Italian Ministry of Environment, Land and Sea.

Edited by: B. Merz

Reviewed by: two anonymous referees

\section{References}

AAVV: Cinquant'anni di dati meteo-climatici in Piemonte, Arpa, available at: http://rsaonline.arpa.piemonte.it/meteoclima50/ intro.htm, last access: 23 April 2013, 2011 (in Italian).

Bates, B. C., Kundzewicz, Z. W., Wu, S., and Palutikof, J. P.: Climate Change and Water, IPCC Secretariat, Geneva, Tech. rep., 210 pp., 2008. 
Beniston, M.: Mountain Climates and Climatic Change: An Overview of Processes Focusing on the European Alps, Pure Appl. Geophys., 162, 1587-1606, doi:10.1007/s00024-0052684-9, 2005

Ciccarelli, N., Von-Hardenberg, J., Provenzale, A., Ronchi, C., Vargiu, A., and Pelosini, R.: Climate variability in north-western Italy during the second half of the 20th century, Global Planet. Change, 63, 185-195, doi:10.1016/j.gloplacha.2008.03.006, 2008.

Flaounas, E., Drobinski, P., Borga, M., Calvet, J., Delrieu, G., Morin, E., Tartari, G., and Toffolon, R.: Assessment of gridded observations used for climate model validation in the Mediterranean region: the HyMeX and MED-CORDEX framework, Environ. Res. Lett., 7, 024017, doi:10.1088/1748-9326/7/2/024017, 2012.

Frei, C. and Schär, C.: A precipitation climatology of the Alps from high-resolution rain-gauge observations, Int. J. Climatol., 18, 873-900, doi:10.1002/(SICI)10970088(19980630)18:8<873::AID-JOC255>3.0.CO;2-9, 1998.

Frei, C., Christensen, J. H., Déqué, M., Jacob, D., Jones, R. G., and Vidale, P. L.: Daily precipitation statistics in regional climate models: Evaluation and intercomparison for the European Alps, J. Geophys. Res., 108, 1-19, doi:10.1029/2002JD002287, 2003.

Haylock, M. R., Hofstra, N., Klein Tank, A. M. G., Klok, E. J., Jones, P. D., and New, M.: A European daily highresolution gridded data set of surface temperature and precipitation for 1950-2006, J. Geophys. Res., 113, D20119, doi:10.1029/2008JD010201, 2008.

Herrera, S., Gutiérrez, J. M., Ancell, R., Pons, M. R., Frías, M. D., and Fernández, J.: Development and analysis of a 50-year high-resolution daily gridded precipitation dataset over Spain (Spain02), Int. J. Climatol., 32, 74-85, doi:10.1002/joc.2256, 2012.

Hofstra, N., Haylock, M., New, M., and Jones, P. D.: Testing EOBS European high-resolution gridded data set of daily precipitation and surface temperature, J. Geophys. Res., 114, D21101, doi:10.1029/2009JD011799, 2009.

Hofstra, N., New, M., and McSweeney, C.: The influence of interpolation and station network density on the distributions and trends of climate variables in gridded daily data, Clim. Dynam., 35, 841-858, doi:10.1007/s00382-009-0698-1, 2010.

Kalnay, E.: Atmospheric modeling, data assimilation, and predictability, Cambridge University Press, Cambridge, UK, New York, 2003.

Kyselý, J. and Plavcová, E.: A critical remark on the applicability of E-OBS European gridded temperature data set for validating control climate simulations, J. Geophys. Res., 115, D23118, doi:10.1029/2010JD014123, 2010.

Maraun, D., Osborn, T., and Rust, H.: The influence of synoptic airflow on UK daily precipitation extremes. Part II: regional climate model and E-OBS data validation, Clim. Dynam., 39, 287-301, doi:10.1007/s00382-011-1176-0, 2012.

Murphy, A.: What is a good forecast? An essay on the nature of goodness in weather forecasting, Weather Forecast., 8, 281-293, doi:10.1175/1520-0434(1993)008<0281:WIAGFA > 2.0.CO;2, 1993.
Ronchi, C., Luigi, C. D., Ciccarelli, N., and Loglisci, N.: Development of a daily gridded climatological air temperature dataset based on a optimal interpolation of ERA-40 reanalysis downscaling and a local high resolution thermometers network, in: 8th EMS Annual Meeting and 7th European Conference on Applied Climatology, Amsterdam, The Netherlands, EMS8/ECAC7 Abstracts, Vol. 5, EMS2008-A-00492, 2008.

Schär, C., Davies, T. D., Frei, C., Wanner, H., Widmann, M., Wild, M., and Davies, H. C.: Current Alpine climate. A View from the Alps: Regional Perspectives on Climate Change, edited by: Cebon, P., Dahinden, U., Davies, H. C., Imboden, D., and Jäger, C., The MIT Press, 1998.

Schmidli, J., Frei, C., and Vidale, P. L.: Downscaling from GCM precipitation: a benchmark for dynamical and statistical downscaling methods, Int. J. Climatol., 26, 679-689, doi:10.1002/joc.1287, 2006.

Schmidli, J., Goodess, C. M., Frei, C., Haylock, M. R., Hundecha, Y., Ribalaygua, J., and Schmith, T.: Statistical and dynamical downscaling of precipitation: An evaluation and comparison of scenarios for the European Alps, J. Geophys. Res., 112, D04105, doi:10.1029/2005JD007026, 2007.

Shepard, D. S.: Computer mapping: the SYMAP interpolation algorithm., in: Spatial Statistics and Models, edited by: Gaile, G. and Willmott, C., Reidel Publishing Company, Dordrecht, Netherlands, 133-145, 1984.

Suklitsch, M., Gobiet, A., Truhetz, H., Awan, N., Göttel, H., and Jacob, D.: Error characteristics of high resolution regional climate models over the Alpine area, Clim. Dynam., 37, 377-390, doi:10.1007/s00382-010-0848-5, 2011.

Taylor, K. E.: Summarizing multiple aspects of model performance in a single diagram, J. Geophys. Res., 106, 7183-7192, doi:10.1029/2000JD900719, 2001.

Turco, M., Quintana-Seguí, P., Llasat, M. C., Herrera, S., and Gutiérrez, J. M.: Testing MOS precipitation downscaling for ENSEMBLES regional climate models over Spain, J. Geophys. Res., 116, D18109, doi:10.1029/2011JD016166, 2011.

Uboldi, F., Lussana, C., and Salvati, M.: Three-dimensional spatial interpolation of surface meteorological observations from high-resolution local networks, Meteorol. Appl., 15, 331-345, doi:10.1002/met.76, 2008 .

WMO: Guidelines on Analysis of extremes in a changing climate in support of informed decisions for adaptation, Tech. Rep. WCDMP No. 72, WMO/TD-No. 1500, WMO, 2009.

Zollo, A. L., Mercogliano, P., Turco, M., Vezzoli, R., Rianna, G., Bucchignani, E., Manzi, M. P., and Montesarchio, M.: Architectures and tools to analyse the impact of climate change on hydrogeological risk on Mediterranean area, Tech. rep., CMCC, available at: http://www.cmcc.it/pubblicazioni/ pubblicazioni/research-papers/rp0129-isc-03-2012.pdf, last access: 23 April 2013, 2012. 